\title{
Representation of Group Isomorphisms: The Compact Case
}

\author{
Marita Ferrer, ${ }^{1}$ Margarita Gary, ${ }^{2}$ and Salvador Hernández ${ }^{3}$ \\ ${ }^{1}$ Universitat Jaume I, IMAC, Campus de Riu Sec, 12071 Castellón, Spain \\ ${ }^{2}$ Departamento de Matemáticas, Universidad Autónoma Metropolitana, Iztapalapa, 14387 México, DF, Mexico \\ ${ }^{3}$ Departamento de Matemáticas, Universitat Jaume I, Campus de Riu Sec, 12071 Castellón, Spain
}

Correspondence should be addressed to Salvador Hernández; hernande@mat.uji.es

Received 31 October 2014; Accepted 12 January 2015

Academic Editor: Hugo Leiva

Copyright (C) 2015 Marita Ferrer et al. This is an open access article distributed under the Creative Commons Attribution License, which permits unrestricted use, distribution, and reproduction in any medium, provided the original work is properly cited.

Let $G$ be a discrete group and let $\mathscr{A}$ and $\mathscr{B}$ be two subgroups of $G$-valued continuous functions defined on two 0 -dimensional compact spaces $X$ and $Y$. A group isomorphism $H$ defined between $\mathscr{A}$ and $\mathscr{B}$ is called separating when, for each pair of maps $f, g \in \mathscr{A}$ satisfying that $f^{-1}\left(e_{G}\right) \cup g^{-1}\left(e_{G}\right)=X$, it holds that $H f^{-1}\left(e_{G}\right) \cup H g^{-1}\left(e_{G}\right)=Y$. We prove that under some mild conditions every biseparating isomorphism $H: \mathscr{A} \rightarrow \mathscr{B}$ can be represented by means of a continuous function $h: Y \rightarrow X$ as a weighted composition operator. As a consequence we establish the equivalence of two subgroups of continuous functions if there is a biseparating isomorphism defined between them.

\section{Introduction}

Let $G$ be a discrete group and let $X$ and $Y$ be topological spaces. If $\mathscr{A}$ and $\mathscr{B}$ are groups of $G$-valued continuous maps, we say that $\mathscr{A}$ and $\mathscr{B}$ are equivalent when there is a homeomorphism $h: Y \rightarrow X$ and a continuous map $\omega: Y \rightarrow \operatorname{Aut}(G)$ such that the map $H: \mathscr{A} \rightarrow \mathscr{B}$ defined as $H f(y)=\omega[y](f(h(y))), f \in \mathscr{A}, y \in Y$, is a group isomorphism of $\mathscr{A}$ onto $\mathscr{B}$. Here $\operatorname{Aut}(G)$ is equipped with the pointwise convergence topology. We say in this case that $H$ is represented as a weighted composition operator. There are many results that are concerned with the representation of linear operators as weighted composition maps and the equivalence of specific groups of continuous functions in the literature, which is vast in this regard. We will only mention here the classic Banach-Stone theorem that, when $G$ is the field of real or complex numbers, establishes that if the Banach spaces of continuous functions $C(X, G)$ and $C(Y, G)$ are isometric, then they are equivalent and the isometry can be represented as a weighted composition map (cf. [1-10]). Another important example appears in coding theory, where the well-known MacWilliams Equivalence Theorem asserts that when $G$ is a finite field and $X$ and $Y$ are finite sets, two codes (linear subspaces) $\mathscr{A}$ and $\mathscr{B}$ of $G^{X}$ and $G^{Y}$, respectively, are equivalent when they are isometric for the Hamming metric (see [11-13]). This result has been generalized to convolutional codes in [14] and it also makes sense in other areas, for example, functional analysis and linear dynamical systems (cf. [14-18]). The main motivation of this research has been to extend MacWilliams Equivalence Theorem to more general settings and explore the possible application of these methods to the study of convolutional codes or linear dynamical systems. However, throughout this paper, we will only deal with 0 -dimensional compact spaces $X$ and $Y$ and a discrete group $G$. We will look at the possible application of this abstract approach elsewhere. There are many precedents in the study of the representation of group homomorphisms for group-valued continuous functions. Among them, the following ones are relevant here (cf. [19-26]). Most basic facts and notions related to topological properties may be found in [27].

Throughout this paper all spaces are assumed to be Hausdorff 0 -dimensional and compact. That is to say, we only deal with Hausdorff compact spaces that contain an open basis consisting of closed and open (clopen) subsets. If $X$ is a topological space and $G$ is a topological (discrete) group, we denote by $C(X, G)$ the group of continuous functions from $X$ to $G$. Let $e_{G}$ be the neutral element of $G$. For $f \in C(X, G)$ 
the cozero of $f$ is the set $\operatorname{coz}(f)=\left\{x \in X: f(x) \neq e_{G}\right\}$ and the zero of $f$ is the set $Z(f)=X \backslash \operatorname{coz}(f)$. Since $G$ is discrete, $\operatorname{coz}(f)$ and $Z(f)$ are both clopen subsets of $X$.

Let $\mathscr{A}$ be a subgroup of $C(X, G)$ and set $Z(\mathscr{A}) \stackrel{\text { def }}{=}\{Z(f)$ : $f \in \mathscr{A}\}$. Then $\sigma(Z(\mathscr{A}))$ denotes the minimum collection of subsets containing $Z(\mathscr{A})$ that is closed under finite unions and intersections (resp., $\operatorname{coz}(\mathscr{A}) \stackrel{\text { def }}{=}\{\operatorname{coz}(f): f \in \mathscr{A}\}$ and $\sigma(\operatorname{coz}(\mathscr{A}))$ denotes the minimum collection of subsets containing $\operatorname{coz}(\mathscr{A})$ that is closed under finite unions and intersections). It is said that $\mathscr{A}$ separates points in $X$ if for every pair of distinct points $\left(x_{1}, x_{2}\right) \in X \times X$ there is a map $f \in \mathscr{A}$ such that $f\left(x_{1}\right) \neq e_{G}$ and $f\left(x_{2}\right)=e_{G}$. It is said that $\mathscr{A}$ strongly separates points in $X$ if, for every pair $\left(x_{1}, x_{2}\right) \in X \times X$, there are maps $f_{1}, f_{2} \in \mathscr{A}$ such that $x_{i} \in \operatorname{coz}\left(f_{i}\right), 1 \leq i \leq 2$, and $\operatorname{coz}\left(f_{1}\right) \cap \operatorname{coz}\left(f_{2}\right)=\emptyset$.

As group $G$ is finite, it could be thought that $\operatorname{coz}(\mathscr{A})$ and $\sigma(\operatorname{coz}(\mathscr{A}))$ should coincide. However, this is misleading as the following example shows. Obviously, by Morgan's laws, it suffices to prove that $Z(\mathscr{A}) \neq \sigma(Z(\mathscr{A}))$.

Example 1. Let $G$ be the group with two elements $\{0,1\}$ and take $X=G^{\mathbb{N}}$ equipped with the product topology. Clearly $X$ is a compact space homeomorphic to the Cantor set, which is 0 -dimensional. Let $p_{m} \in C(X, G)$ be the $m$ th projection on $X$ and set $\mathscr{A}$ as the subgroup of $C(X, G)$ generated the collection $\left\{p_{m}: m \in \mathbb{N}\right\}$. Take $n \in \mathbb{N}$ greater than 2 and $H_{n} \stackrel{\text { def }}{=}\left\{\left(x_{j}\right) \in\right.$ $\left.X: x_{1}=\cdots=x_{n}=0\right\}$. It is easily verified that $H_{n}$ belongs to $\sigma(Z(\mathscr{A}))$. However, take an arbitrary but fixed element $f \in$ $\mathscr{A}$. Denote by $e_{k}$ the element in $X$ such that $p_{m}\left(e_{k}\right)=0$ if $m \neq k$ and $p_{k}\left(e_{k}\right)=1$ and $\operatorname{set} \sup (f) \stackrel{\text { def }}{=}\left\{k \in \mathbb{N}: f\left(e_{k}\right)=1\right\}$. If $\{1, \ldots, n\} \subsetneq \sup (f)$, then there is $l<n$ such that $l \notin \sup (f)$. Therefore $f\left(e_{l}\right)=0$, which yields $e_{l} \in Z(f) \backslash H_{n}$. On the other hand, if $\{1, \ldots, n\} \subseteq \sup (f)$, take $1 \leq n_{1}<n_{2} \leq n$. We have that $f\left(e_{n_{1}}+e_{n_{2}}\right)=0$, which again yields $e_{n_{1}}+e_{n_{2}} \in$ $Z(f) \backslash H_{n}$. In either case, we obtain that $Z(f) \neq H_{n}$. Thus $H_{n} \in \sigma(Z(\mathscr{A})) \backslash Z(\mathscr{A})$, which completes the proof.

Denote by $\delta_{x}: \mathscr{A} \rightarrow G$ the evaluation map; that is, $\delta_{x}(f)=f(x)$ for every $f \in \mathscr{A}$. It is said that $\mathscr{A}$ is pointwise dense when $\delta_{x}(\mathscr{A})$ is dense in $G$ for all $x \in X$. It is said that $\mathscr{A} \subseteq C(X, G)$ is controllable if for every $f \in \mathscr{A}$ and $D_{1}, D_{2} \in \sigma(Z(\mathscr{A}))$ such that $D_{1} \cap D_{2}=\emptyset$ there exist a subset $U \subseteq \sigma(\operatorname{coz}(\mathscr{A}))$ and a function $g \in \mathscr{A}$ such that $D_{1} \subseteq U \subseteq X \backslash D_{2},\left.g\right|_{D_{1}}=\left.f\right|_{D_{1}}$, and $\left.g\right|_{Z(f) \cup(X \backslash U)} \equiv e_{G}$.

We now formulate our main results.

Theorem 2. Let $X$ and $Y$ be 0-dimensional compact Hausdorff spaces and let $G$ be a discrete group. Suppose that $\mathscr{A}$ and $\mathscr{B}$ are controllable and pointwise dense subgroups of $G$ valued continuous functions separating the points of $X$ and $Y$, respectively. If $H: \mathscr{A} \rightarrow \mathscr{B}$ is a biseparating group isomorphism of $\mathscr{A}$ onto $\mathscr{B}$, then there are continuous maps $h: Y \rightarrow X$ and $\omega: Y \rightarrow$ Aut $(G)$ satisfying the following properties.

(1) $h$ is a homeomorphism of $Y$ onto $X$.

(2) For each $y \in Y$ and every $f \in \mathscr{A}$ it holds

$$
H f(y)=\omega[y](f(h(y))) .
$$

(3) $H$ is a continuous isomorphism with respect to the pointwise convergence topology.

(4) $H$ is a continuous isomorphism with respect to the compact open topology.

Corollary 3. Let $X$ and $Y$ be 0-dimensional compact Hausdorff spaces and let $G$ be a discrete group. Suppose that $\mathscr{A}$ and $\mathscr{B}$ are controllable and pointwise dense subgroups of $G-$ valued continuous functions separating the points of $X$ and $Y$, respectively. If there is a biseparating group isomorphism $\mathrm{H}$ of $\mathscr{A}$ onto $\mathscr{B}$, then $\mathscr{A}$ and $\mathscr{B}$ are equivalent.

We notice that some of the requirements we have imposed on the previous results could be relaxed in general. However, this would take us to a wider setting in general. For instance, if we assume that $\mathscr{A}$ does not separate points in $X$, then there must be some point $x \in X$ such that $f(x)=e_{G}$ for all $f \in \mathscr{A}$; then we can replace $X$ by the largest subspace $X_{\mathscr{A}} \subseteq X$ where $\mathscr{A}$ separates points. This subspace $X_{\mathscr{A}}$ is open but not necessarily closed in general. Thus, the study of subgroups that does not separate points leads us to consider locally compact spaces. We will discuss these spaces in a subsequent paper.

\section{Basic Notions and Facts}

The following lemma is easily verified using a standard compactness argument. Recall that we are assuming that all spaces are compact and 0-dimensional.

Lemma 4. Let $D$ be a family of clopen subsets of $X$ that is a subbase for the closed subsets of $X$. Then for every disjoint nonempty closed subsets $A$ and $B$ of $X$ there are two disjoint subsets $D_{A}$ and $D_{B}$ in $\sigma(D)$ such that $A \subseteq D_{A}$ and $B \subseteq D_{B}$.

Example 5. A specific example where Lemma 4 applies is given when $\mathscr{A}$ separates points in $X$, where $\mathscr{D}=Z(\mathscr{A})$.

Next proposition shows that the notions of separating and strongly separating points are equivalent for controllable subgroups.

Proposition 6. If $\mathscr{A}$ is a controllable subgroup of $C(X, G)$ that separates the points of $X$, then $\mathscr{A}$ strongly separates the points of $X$.

Proof. Set $\mathscr{D}=Z(\mathscr{A})$ and take two distinct elements $x_{1} \neq x_{2}$ in $X$. Applying Lemma 4 , since $\mathscr{A}$ separates the points of $X$, there are $D_{1}, D_{2} \in \sigma(\mathscr{D})$ such that $x_{1} \in D_{1}, x_{2} \in D_{2}$, and $D_{1} \cap D_{2}=\emptyset$. Take $f_{i} \in \mathscr{A}$ such that $f_{i}\left(x_{i}\right) \neq e_{G}, i \in\{1,2\}$. Since $\mathscr{A}$ is controllable, we have $U_{1} \in \sigma(\operatorname{coz}(\mathscr{A}))$ and $g_{1} \in \mathscr{A}$ such that $D_{1} \subseteq U_{1} \subseteq X \backslash D_{2},\left.g_{1}\right|_{D_{1}}=\left.f_{1}\right|_{D_{1}}$, and $Z\left(f_{1}\right) \cup(X \backslash$ $\left.U_{1}\right) \subseteq Z\left(g_{1}\right)$. Therefore $x_{1} \in \operatorname{coz}\left(g_{1}\right) \subseteq U_{1}$.

Applying the fact that $\mathscr{D}$ is a subbase of closed subsets and using a compactness argument, we deduce that there is $D_{1}^{\prime} \in \sigma(\mathscr{D})$ such that $U_{1} \subseteq D_{1}^{\prime}$ and $D_{1}^{\prime} \cap D_{2}=\emptyset$. By the controllability of $\mathscr{A}$ again, we have $U_{2} \in \sigma(\operatorname{coz}(\mathscr{A}))$ and $g_{2} \in$ $\mathscr{A}$ such that $D_{2} \subseteq U_{2} \subseteq X \backslash D_{1}^{\prime},\left.g_{2}\right|_{D_{2}}=\left.f_{2}\right|_{D_{2}}$, and $Z\left(f_{2}\right) \cup$ $\left(X \backslash U_{2}\right) \subseteq Z\left(g_{2}\right)$. Therefore $x_{2} \in \operatorname{coz}\left(g_{2}\right) \subseteq U_{2}$, which yields $\operatorname{coz}\left(g_{1}\right) \cap \operatorname{coz}\left(g_{2}\right)=\emptyset$. This completes the proof. 
Definition 7. Let $\mathscr{A}$ be a subgroup of $C(X, G)$ and let $\varphi: \mathscr{A} \rightarrow$ $G$ be a group homomorphism. A subset $A$ of $X$ is said to be a support for $\varphi$ if, given $f \in \mathscr{A}$ with $A \subseteq Z(f)$, it holds that $\varphi(f)=e_{G}$.

Some basic properties of support subsets are shown in the next proposition. Observe that since $A \subseteq \bar{A}^{X} \subseteq Z(f)$, we may assume without loss of generality that all support subsets are closed and therefore compact subsets of $X$.

Proposition 8. Let $\varphi: \mathscr{A} \rightarrow G$ be a nonnull group homomorphism. The following assertions hold.

(1) $X$ is a support for $\varphi$.

(2) If $A$ is a support for $\varphi$ then $A \neq \emptyset$.

(3) If $A$ is a support for $\varphi$ and $A \subseteq B$ then $B$ is a support for $\varphi$.

(4) Let $A$ be a support for $\varphi$ and $f, g \in \mathscr{A}$ such that $\left.f\right|_{A}=$ $\left.g\right|_{A}$. Then $\varphi(f)=\varphi(g)$.

If, in addition, $\mathscr{A}$ is controllable and separates points in $X$, then we have the following.

(5) Let $A$ and $B$ be supports for $\varphi$; then $A \cap B \neq \emptyset$.

Proof. Assertions (1)-(4) are obvious.

(5) Let $A$ and $B$ be closed supports for $\varphi$. Suppose $A \cap$ $B=\emptyset$. Since $\mathscr{A}$ separates points in $X$, by Lemma 4 and Proposition 6 , there are two disjoint subsets $D_{A}$ and $D_{B}$ in $\sigma(Z(\mathscr{A}))$ containing $A$ and $B$, respectively. Take $f \in \mathscr{A}$ such that $\varphi(f) \neq e_{G}$. Applying the controllability of $\mathscr{A}$, we obtain $U \in \sigma(\operatorname{coz}(\mathscr{A}))$ and $g \in \mathscr{A}$ such that $A \subseteq D_{A} \subseteq U \subseteq$ $X \backslash D_{B} \subseteq X \backslash B,\left.g\right|_{D_{A}}=\left.f\right|_{D_{A}}$, and $Z(f) \cup(X \backslash U) \subseteq Z(g)$. This yields a contradiction as the evaluation of $\varphi(g)$ shows. Indeed, since $g(x)=f(x)$ for all $x \in A$, by item (4) it follows that $\varphi(g)=\varphi(f) \neq e_{G}$. On the other hand, we have that $g(x)=e_{G}$ for all $x \in B$, which imples $\varphi(g)=e_{G}$. This contradiction completes the proof.

Definition 9. A map $H: \mathscr{A} \rightarrow \mathscr{B}$ is said to be separating or disjointness preserving, if, for each pair of maps $f, g \in \mathscr{A}$ satisfying that $f^{-1}\left(e_{G}\right) \cup g^{-1}\left(e_{G}\right)=X$, it holds that $H f^{-1}\left(e_{G}\right) \cup$ $\mathrm{Hg}^{-1}\left(e_{G}\right)=Y$ (equivalently, if $\operatorname{coz}(f) \cap \operatorname{coz}(g)=\emptyset$ implies $\operatorname{coz}(H f) \cap \operatorname{coz}(H g)=\emptyset$ for all $f, g \in \mathscr{A})$. In case $H$ is bijective, the map $H$ is said to be biseparating if both $H$ and $H^{-1}$ are separating. Remark that this definition makes sense and extends naturally to maps $\varphi: \mathscr{A} \rightarrow G$.

Remark 10. Originally, separating maps for scalar-valued continuous functions were defined as those maps $H$ such that $f g=0$ implies $\mathrm{HfHg}=0$. If one interprets the null element 0 as the identity $e$ of the group, then separating maps could be defined as those maps that satisfy $f(x) g(x)=e$ for all $x \in X$ which implies $H f(y) H g(y)=e$ for all $y \in Y$ or that $H\left(f^{-1}\right)=$ $H(f)^{-1}$ for all $f \in \mathscr{A}$. Obviously this definition would be vacuous here since we are assuming that $H$ is a group homomorphism throughout this paper. Thus, this definition would take us to the more general question of representing group isomorphisms defined between groups of continuous functions without any further requirement. Unfortunately, this is not possible in general. Indeed, a remarkable result due to Milutin [28] establishes that if $K$ is an uncountable compact metric space, then $C(K, \mathbb{C})$ is linearly isomorphic to $C([0,1], \mathbb{C})$. Therefore, it is essential to impose some extra algebraic or geometrical condition on the isomorphisms $H$ in order to be able to represent them by continuous maps defined on the compact spaces $K$ and $[0,1]$. In this sense, the connection with separating isomorphisms stems from [8], where it was proved that every linear isometry is a separating isomorphism.

Next we will see that every nonnull separating group homomorphism $\varphi: \mathscr{A} \rightarrow G$, where $\mathscr{A}$ is controllable, has the smallest possible compact support set. For that purpose, set

$$
\mathcal{S}=\{A \subseteq X: A \text { is a compact support for } \varphi\} .
$$

There is a canonical partial order that can be defined on $\mathcal{S}$ : $A \leq B, A, B \in \mathcal{S}$, if and only if $B \subseteq A$. A standard argument shows that $(\mathcal{S}, \leq)$ is an inductive set and, by Zorn's lemma, $\mathcal{S}$ contains a $\subseteq$-minimal element. Furthermore, this minimal element is in fact a minimum because of the next proposition.

Proposition 11. If $A$ separates points in $X$, then the minimum element $\mathcal{S}$ consists of a singleton.

Proof. Let $S$ be a minimal element of $\delta$, which is nonempty by Proposition 8 . Suppose now that there are two different elements $x_{1}, x_{2}$ that are contained in $S$. As $X$ is Hausdorff, we can select two disjoint open subsets $V_{1}, V_{2}$ in $X$ such that $x_{1} \in V_{1}$ and $x_{2} \in V_{2}$. Since $S$ is minimal, the compact subset $S \backslash V_{i}$ is not a support for $\varphi$. Hence, there are $f_{i} \in \mathscr{A}$ such that $S \backslash V_{i} \subseteq Z\left(f_{i}\right)$ and $\varphi\left(f_{i}\right) \neq e_{G}, 1 \leq i \leq 2$. Since $\varphi$ is separating, it follows that $A=\operatorname{coz}\left(f_{1}\right) \cap \operatorname{coz}\left(f_{2}\right)$ is a nonempty compact subset of $X$.

We claim that $S \cap A=\emptyset$. Otherwise, pick up an element $a \in S \cap A$. If $a \in V_{1}$ then $a \in S \backslash V_{2}$ and $a \in Z\left(f_{2}\right)$, which is a contradiction; but if $a \notin V_{1}$ then $a \in S \backslash V_{1}$, which implies that $a \in Z\left(f_{1}\right)$ and we get a contradiction again. Therefore $S \cap A=\emptyset$. By Lemma 4 , we can take two disjoint sets $D_{S}, D_{A} \in$ $\sigma(Z(\mathscr{A}))$ such that $S \subseteq D_{S}$ and $A \subseteq D_{A}$. Applying the fact that $\mathscr{A}$ is controllable to $D_{S}, D_{A}$ and $f_{1}$, we obtain a set $U \in$ $\sigma(\operatorname{coz}(\mathscr{A}))$ and a map $g \in \mathscr{A}$ such that $S \subseteq D_{S} \subseteq U \subseteq X \backslash D_{A} \subseteq$ $X \backslash A,\left.g\right|_{S}=\left.f_{1}\right|_{S}$, and $\left.g\right|_{Z\left(f_{1}\right) \cup(X \backslash U)} \equiv e_{G}$. Then $U \cap A=\emptyset$, $\varphi(g)=\varphi\left(f_{1}\right) \neq e_{G}$, and $A \subseteq Z(g)$. Since $\varphi$ is separating the set $B=\operatorname{coz}(g) \cap \operatorname{coz}\left(f_{2}\right) \neq \emptyset$, take $b \in B$. Then $b \in \operatorname{coz}\left(f_{2}\right)$ and $b \in \operatorname{coz}(g) \subseteq \operatorname{coz}\left(f_{1}\right)$; that is, $b \in \operatorname{coz}\left(f_{1}\right) \cap \operatorname{coz}\left(f_{2}\right)=A$. As a consequence $g(b)=e_{G}$, which is a contradiction. Therefore we have proved that $|S|=1$. This completes the proof.

\section{Proof of Main Results}

Along this section $\mathscr{A}$ (resp., $\mathscr{B}$ ) is a controllable subgroup of $C(X, G)$ (resp., $C(Y, G)$ ) that separates points in $X$ (resp., $Y$ ).

Let $H: \mathscr{A} \rightarrow \mathscr{B}$ be a separating group homomorphism. The maps $\delta_{y} \circ H$ are separating group homomorphisms of $\mathscr{A}$ into $G$ for all $y \in Y$. Furthermore, since $\mathscr{A}$ is controllable and separates points in $X$, we can apply Proposition 11, in 
order to obtain that each partial ordered set $\mathcal{S}_{y}=\{A \subseteq X$ : $A$ is a compact support for $\left.\delta_{y} \circ H\right\}$ has a minimum element, which is a singleton denoted by $h(y)$. Therefore, by sending $y \in Y$ to $h(y) \in X$ for every $y \in Y$, we have defined the support map of $Y$ into $X$ that is associated with $H$.

Proposition 12. Let $H: \mathscr{A} \rightarrow \mathscr{B}$ be a separating group homomorphism. Then the support map $h$ has the following properties.

(1) $h$ is continuous.

(2) If $\emptyset \neq A \subseteq X$ is open, $f \in \mathscr{A}$, and $A \subseteq Z(f)$, then $h^{-1}(A) \subseteq Z(H f)$.

(3) $h(\operatorname{coz}(H f)) \subseteq \operatorname{coz}(f)$.

(4) If $H$ is one-to-one, then $h$ is onto.

Moreover, when $H$ is a bijection of $\mathscr{A}$ onto $\mathscr{B}$, we have, in addition, the following.

(5) If $H$ is biseparating, then $h$ is a homeomorphism of $Y$ onto $X$.

Proof. (1) Let $\left(y_{d}\right)_{d \in D}$ be a net in $Y$ converging to $y \in Y$. By a standard compactness argument, we may assume without loss of generality that $\left(h\left(y_{d}\right)\right)_{d}$ converges to $x \in X$. Reasoning by contradiction, suppose $h(y) \neq x$. Since $X$ is Hausdorff, we can take two disjoint open neighborhoods $V_{h(y)}$ and $V_{x}$ of $h(y)$ and $x$, respectively. Using convergence, there is $d_{1} \in D$ such that $h\left(y_{d}\right) \in V_{x}$ for all $d \geq d_{1}$.

As every support subset for $\delta_{y^{\prime}} \circ H$ contains $h\left(y^{\prime}\right)$, for all $y^{\prime} \in Y$, the subset $X \backslash V_{h(y)}$ may not be a support for $\delta_{y} \circ H$. Therefore there exists $f \in \mathscr{A}$ such that $X \backslash V_{h(y)} \subseteq Z(f)$ and $H f(y) \neq e_{G}$. Moreover, since $H(f)$ is a continuous function, the net $\left(H f\left(y_{d}\right)\right)_{d}$ converges to $H f(y)$ and, because $G$ is discrete, there is $d_{2} \geq d_{1}$ such that $H f\left(y_{d}\right) \neq e_{G}$ for all $d \geq d_{2}$. If we take and index $d_{3} \in D$ such that $d_{3} \geq d_{2}$, then the subset $X \backslash V_{x}$ may not be a support for $\delta_{y_{d_{3}}} \circ H$. Thus, there exists $f_{3} \in \mathscr{A}$ such that $X \backslash V_{x} \subseteq Z\left(f_{3}\right)$ and $H f_{3}\left(y_{d_{3}}\right) \neq e_{G}$. This means that $y_{d_{3}} \in \operatorname{coz}\left(H f_{3}\right) \cap \operatorname{coz}(H f)$ and, since $H$ is a separating map, it follows that $\operatorname{coz}\left(f_{3}\right) \cap \operatorname{coz}(f) \neq \emptyset$. But $\operatorname{coz}\left(f_{3}\right) \subseteq V_{x}$, which is disjoint from $\operatorname{coz}(f) \subseteq V_{h(y)}$. This is a contradiction that completes the proof.

(2) Let $\emptyset \neq A \subsetneq X$ be an open subset, $f \in \mathscr{A}$, and $A \subseteq$ $Z(f)$. If we take $y \in h^{-1}(A)$, then $X \backslash A$ is a nonempty compact subset that is not a support for $\delta_{y} \circ H$. Then there is $g \in \mathscr{A}$ such that $X \backslash A \subseteq Z(g)$ and $H g(y) \neq e_{G}$. Therefore $\operatorname{coz}(g) \subseteq$ $A$ and $\operatorname{coz}(f) \subseteq X \backslash A$. Since $H$ is separating, we have that $\operatorname{coz}(H g) \cap \operatorname{coz}(H f)=\emptyset$. Therefore $H f(y)=e_{G}$.

(3) Take $x \in h(\operatorname{coz}(H f))$. Then $x=h(y)$ for some $y \in$ $\operatorname{coz}(H f)$. Since $h(y)$ is a support for $\delta_{y} \circ H$, it follows that $x \notin Z(f)$ or, equivalently, we have $x \in \operatorname{coz}(f)$.

(4) Suppose $h(Y) \neq X$ and take $x \in X$ such that $x \notin h(Y)$. Since $h$ is continuous and $Y$ is compact, we have that $h(Y)$ is a proper compact subset of $X$. Applying Lemma 4, there are two disjoint subsets $D_{x}, D_{Y} \in \sigma(Z(\mathscr{A}))$ such that $x \in D_{x}$ and $h(Y) \subseteq D_{Y}$. Moreover, as $\mathscr{A}$ separates points in $X$, there exists $f \in \mathscr{A}$ such that $f(x) \neq e_{G}$. Again, by the controllability of $\mathscr{A}$, we may take a subset $U \subseteq \sigma(\operatorname{coz}(\mathscr{A}))$ and a map $g \in \mathscr{A}$ such that $x \in D_{x} \subseteq U \subseteq X \backslash D_{Y} \subseteq X \backslash h(Y),\left.g\right|_{D_{x}}=\left.f\right|_{D_{x}}$, and
$Z(f) \cup(X \backslash U) \subseteq Z(g)$. As a consequence $g(x)=f(x) \neq e_{G}$, $h(Y) \subseteq Z(g)$, and $H(f)(y)=e_{G}$ for all $y \in Y$. Then $H f \equiv e_{G}$. Since $H$ is an injective group homomorphism, this yields $f \equiv$ $e_{G}$, which is a contradiction.

(5) Since $X$ and $Y$ are compact spaces, it will suffice to prove that $h$ is one-to-one. Suppose there are two elements $y_{1} \neq y_{2}$ in $Y$ such that $h\left(y_{1}\right)=h\left(y_{2}\right)=x_{0}$. Since $\mathscr{B}$ separates the points of $Y$, there are $D_{1}^{\prime}, D_{2}^{\prime} \in \sigma(Z(\mathscr{B}))$ such that $y_{1} \in$ $D_{1}^{\prime}, y_{2} \in D_{2}^{\prime}$, and $D_{1}^{\prime} \cap D_{2}^{\prime}=\emptyset$. Since $H(\mathscr{A})=\mathscr{B}$, there is $f_{i} \in \mathscr{A}$ such that $H\left(f_{i}\right)\left(y_{i}\right) \neq e_{G}$, for $i \in\{1,2\}$. Since $\mathscr{B}$ is controllable, there are $U_{1} \in \sigma(\operatorname{coz}(\mathscr{B})), g_{1} \in \mathscr{A}$ such that $y_{1} \in$ $D_{1}^{\prime} \subseteq U_{1} \subseteq Y \backslash D_{2}^{\prime},\left.H g_{1}\right|_{D_{1}^{\prime}}=\left.H f_{1}\right|_{D_{1}^{\prime}}$, and $\left.H g_{1}\right|_{Z\left(H f_{1}\right) \cup\left(Y \backslash U_{1}\right)} \equiv$ $e_{G}$. Since $D_{2}^{\prime} \cap U_{1}=\emptyset$, applying a compactness argument, there is $D_{1}^{\prime \prime} \in \sigma(Z(\mathscr{A}))$ such that $U_{1} \subseteq D_{1}^{\prime \prime}$ and $D_{2}^{\prime} \cap D_{1}^{\prime \prime}=\emptyset$. Now, by the controllability of $\mathscr{B}$, there are $U_{2} \in \sigma(\operatorname{coz}(\mathscr{B}))$ and $g_{2} \in \mathscr{A}$ such that $y_{2} \in D_{2}^{\prime} \subseteq U_{2} \subseteq Y \backslash D_{1}^{\prime \prime},\left.H g_{2}\right|_{D_{2}^{\prime}}=$ $\left.H f_{2}\right|_{D_{2}^{\prime}}$, and $\left.H g_{2}\right|_{Z\left(H f_{2}\right) \cup\left(Y \backslash U_{2}\right)} \equiv e_{G}$. Hence, since $\operatorname{coz}\left(H g_{i}\right) \subseteq$ $U_{i}, U_{1} \cap U_{2}=\emptyset$, and $H$ is biseparating, it follows that $\operatorname{coz}\left(g_{1}\right) \cap$ $\operatorname{coz}\left(g_{2}\right)=\emptyset$. On the other hand $H_{i}\left(y_{i}\right)=H f_{i}\left(y_{i}\right) \neq e_{G}$, $i \in\{1,2\}$, and by item (3) above, we have that $h\left(y_{1}\right)=h\left(y_{2}\right)=$ $x_{0} \in \operatorname{coz}\left(g_{1}\right) \cap \operatorname{coz}\left(g_{2}\right)$, which is a contradiction.

We have just seen how a separating group homomorphism $H$ has associated a continuous map $h$ that assigns to each point $y \in Y$ the support subset of $\delta_{y} \circ H$. Our next goal now is to obtain a complete representation of $H$ by means of the support map $h$. Having this in mind, set

$$
G_{h(y)} \stackrel{\text { def }}{=} \operatorname{Im}\left(\delta_{h(y)}\right)=\{f(h(y)): f \in \mathscr{A}\}
$$

which is a subgroup of $G$ for all $y \in Y$, and denote by Hom $\left(G_{h(y)}, G_{y}\right)$ the set of all group homomorphisms on $G_{h(y)}$ into $G_{y}$. Consider now the set

$$
\mathscr{G} \stackrel{\text { def }}{=} \bigcup_{y \in Y} \operatorname{Hom}\left(G_{h(y)}, G_{y}\right) \text {. }
$$

We can think of the elements of $\mathscr{G}$ as partial functions on $G$. That is, functions $\alpha$ : $\operatorname{Dom}(\alpha) \subseteq G \rightarrow G$ whose domain is a (not necessarily proper) subset of $G$. Since the group $G$ is discrete, we can equip $\mathscr{G}$ with the product (or pointwise convergence) topology as follows.

Let $\left[\alpha ; g_{1}, \ldots, g_{n}\right] \stackrel{\text { def }}{=}\left\{\beta \in G^{G}: \alpha\left(g_{i}\right)=\beta\left(g_{i}\right), g_{i} \in\right.$ $G, 1 \leq i \leq n\}$ be a basic neighborhood of a map $\alpha \epsilon$ $G^{G}$. If now $\alpha$ is a partial map, we can restrict this basic neighborhood to $\mathscr{G}$ by letting $\left[\alpha ; g_{1}, \ldots, g_{n}\right]$ be the set of all partial maps $\beta$ : $\operatorname{Dom}(\beta) \subseteq G \rightarrow G$ such that $g_{1}, \ldots, g_{n} \in$ $\operatorname{Dom}(\beta)$ and $\alpha\left(g_{i}\right)=\beta\left(g_{i}\right), 1 \leq i \leq n$. It is easily verified that this procedure extends the pointwise convergence topology on $\mathscr{G}$ (cf. [29]).

With this notation, we define $\omega: Y \rightarrow \mathscr{G}$ by

$$
\omega[y](f(h(y))) \stackrel{\text { def }}{=} H f(y)
$$

for each $y \in Y$. We will see next that $\omega$ is well defined and continuous. 
Proposition 13. With the terminology established above, the following assertions are true.

(1) $\omega[y]$ is a well-defined group homomorphism of $G_{h(y)}$ into $G_{y}$ for all $y \in Y$.

(2) $\omega$ is continuous when $\mathscr{G}$ is equipped with the pointwise convergence topology.

Proof. (1) In order to prove that $\omega[y]$ is well defined, take $f_{1}, f_{2} \in \mathscr{A}$ such that $f_{1}(h(y))=f_{2}(h(y))$. By Proposition 8, we have $\omega[y]\left(f_{1}(h(y))\right)=H f_{1}(y)=H f_{2}(y)=$ $\omega[y]\left(f_{2}(h(y))\right)$. The verification that $\omega[y]$ is a group homomorphism is easy and it is left to the reader.

(2) Let $\left(y_{d}\right)_{d \in D}$ be a net converging to $y$ in $Y$. If $g$ is an arbitrary element in Dom $(\omega[y])=G_{h(y)}$, then $g=f(h(y)) \epsilon$ $G_{h(y)}$ for some $f \in \mathscr{A}$. Since $h$ continuous by Proposition 12, $f \circ h \in C(Y, G)$ and $(f \circ h)^{-1}(g)$ is a clopen neighborhood of $y \in Y$. Since $G$ is discrete, there is $d_{1}(g) \in D$ such that $f\left(h\left(y_{d}\right)\right)=g$ for all $d \geq d_{1}(g)$. Thus $g \in \operatorname{Dom}\left(\omega\left[y_{d}\right]\right)$ for all $d \geq d_{1}(g)$. In like manner, as $\omega[y](g)=H f(y)=g_{y} \epsilon$ $G$ and $H f \in C(Y, G)$, we have that $(H f)^{-1}\left(g_{y}\right)$ is a clopen neighborhood of $y$. As a consequence there is $d_{2} \geq d_{1}(g)$ such that $H f\left(y_{d}\right)=g_{y}$ for all $d \geq d_{2}$. Thus $\omega\left[y_{d}\right](g)=H f\left(y_{d}\right)=$ $g_{y}=H f(y)=\omega[y](g)$ for all $d \geq d_{2}$. This means that the net $\left(\omega\left[y_{d}\right]\right)_{d \in D}$ converges to $\omega[y]$ in the pointwise convergence topology over $\mathscr{G}$.

Observe that since $G$ is discrete, the compact subsets in $G$ are all finite. Therefore, we have also proved that $\omega$ is also continuous if we consider the compact open topology on $\mathscr{G}$. We are in position now of establishing a main result in this paper.

Theorem 14. Let $H: \mathscr{A} \rightarrow \mathscr{B}$ be a separating group homomorphism. Then there are continuous maps

$$
\begin{gathered}
h: Y \longrightarrow X \\
\omega: Y \longrightarrow \bigcup_{y \in Y} \operatorname{Hom}\left(G_{h(y)}, G_{y}\right)
\end{gathered}
$$

satisfying the following properties.

(1) For each $y \in Y$ and every $f \in \mathscr{A}$ it holds

$$
H f(y)=\omega[y](f(h(y))) .
$$

(2) $H$ is continuous with respect to the pointwise convergence topology.

(3) $H$ is continuous with respect to the compact open topology.

(4) If $H$ is biseparating bijection of $\mathscr{A}$ onto $\mathscr{B}$, then $h$ is a homeomorphism.

Proof. Item (1) is consequence of the definition of $\omega$ and (2) follows from assertion (2) in Proposition 13. Thus, only (3) needs to be verified.

Let $\left(f_{d}\right)_{d \in D} \in \mathscr{A}$ be a net converging to $e_{G}$ in the compact open topology. If $K$ is a compact subset of $Y$, then $h(K)$ is a compact subset in $X$ by the continuity of $h$. Therefore $\left(f_{d}\right)_{d}$ is eventually the constant function $e_{G}$ on $h(K)$. Applying (1), it follows that $\left(H f_{d}\right)_{d \in D}$ is eventually $e_{G}$ on $K$, which completes the proof.

Corollary 15. Let $H: \mathscr{A} \rightarrow \mathscr{B}$ be a separating group homomorphism, where $\mathscr{A}$ is pointwise dense. Then there are continuous maps $h: Y \rightarrow X$ and $\omega: Y \rightarrow$ End $(G)$ satisfying the following properties.

(1) For each $y \in Y$ and every $f \in \mathscr{A}$ it holds

$$
H f(y)=\omega[y](f(h(y))) \text {. }
$$

(2) $H$ is continuous with respect to the pointwise convergence topology.

(3) $H$ is continuous with respect to the compact open topology.

(4) If $H$ is a biseparating bijection of $\mathscr{A}$ onto $\mathscr{B}$, then $h$ is a homeomorphism.

We are now in position of establishing the results formulated at Introduction.

Proof of Theorem 2. After Theorem 14 and Corollary 15, we only need to verify that $\omega[y] \in \operatorname{Aut}(G)$ for all $y \in Y$. Applying Corollary 15 to $H^{-1}$, we obtain maps

$$
\begin{gathered}
\rho: X \longrightarrow \operatorname{End}(G), \\
k: X \longrightarrow Y
\end{gathered}
$$

such that for every $x \in X$ and $g \in \mathscr{B}$, we have

$$
H^{-1} g(x)=\rho[x](g(k(x))) .
$$

Thus, for every $f \in \mathscr{A}$ and $x \in X$, we have

$$
\begin{aligned}
f(x) & =H^{-1} \circ(H f)(x)=\rho[x](H f(k(x))) \\
& =\rho[x](\omega[k(x)](f(h(k(x)))))
\end{aligned}
$$

which means that the support subset of $\delta_{x} \circ\left(H^{-1} \circ H\right)$ is both $x$ and $h(k(x))$. Since the minimum support is unique, this means that $h \circ k=i d_{X}$, which implies that $k$ is a right inverse of $h$. Analogously, for every $g \in \mathscr{B}$ and $y \in Y$, we have

$$
\begin{aligned}
g(y) & =H \circ\left(H^{-1} g\right)(y)=\omega[y]\left(H^{-1}(g(h(y)))\right) \\
& =\omega[y](\rho[h(y)](g(k(h(y)))))
\end{aligned}
$$

which means that the support subset of $\delta_{y} \circ\left(H \circ H^{-1}\right)$ is both $y$ and $k(h(y))$. Again, this implies that $k$ is a left inverse of $h$. Since $k$ is both a left and right inverse of $h$, it follows that $k=h^{-1}$. Therefore

$$
\begin{aligned}
f(x) & =H^{-1} \circ(H f)(x)=\rho[x](H f(k(x))) \\
& =\rho[x](\omega[k(x)](f(x))), \\
g(y) & =H \circ\left(H^{-1} g\right)(y)=\omega[y]\left(H^{-1}(g(h(y)))\right) \\
& =\omega[y](\rho[h(y)](g(y))) .
\end{aligned}
$$


Applying the former equality to $x=h(y)$, it follows that $\rho[h(y)] \circ \omega[y]=i d_{G}$ for all $y \in Y$, and from the latter, we also have that $\omega[y] \circ \rho[h(y)]=i d_{G}$. This means that $\omega[y]$ has left and right inverse and, therefore, it is an automorphism on $G$, which completes the proof.

Proof of Corollary 3. It follows directly from Theorem 2.

\section{Conflict of Interests}

The authors declare that there is no conflict of interests regarding the publication of this paper.

\section{Acknowledgments}

The authors thank the referee for several valuable comments. The first and third listed authors acknowledge the partial support provided by the Generalitat Valenciana, Grant code: PROMETEO/2014/062, and by Universitat Jaume I, Grant P1.1B2012-05.

\section{References}

[1] J. Araujo and K. Jarosz, "Biseparating maps between operator algebras," Journal of Mathematical Analysis and Applications, vol. 282, no. 1, pp. 48-55, 2003.

[2] S. Banach, Théorie des opérations linéaires, American Mathematical Society, London, UK, 1955.

[3] J. J. Font and S. Hernandez, "On separating maps between locally compact spaces," Archiv der Mathematik, vol. 63, no. 2, pp. 158-165, 1994.

[4] J. J. Font and S. Hernández, "Automatic continuity and representation of certain linear isomorphisms between group algebras," Indagationes Mathematicae, vol. 6, no. 4, pp. 397-409, 1995.

[5] H.-L. Gau, J.-S. Jeang, and N.-C. Wong, "An algebraic approach to the Banach-Stone theorem for separating linear bijections," Taiwanese Journal of Mathematics, vol. 6, no. 3, pp. 399-403, 2002.

[6] F. González and V. V. Uspenskij, "On homomorphisms of groups of integer-valued functions," Extracta Mathematicae, vol. 14, no. 1, pp. 19-29, 1999.

[7] S. Hernandez, "Uniformly continuous mappings defined by isometries of spaces of bounded uniformly continuous functions," Houston Journal of Mathematics, vol. 29, no. 1, pp. 149155, 2003.

[8] S. Hernández, E. Beckenstein, and L. Narici, "Banach-Stone theorems and separating maps," Manuscripta Mathematica, vol. 86, no. 4, pp. 409-416, 1995.

[9] K. Jarosz, "Automatic continuity of separating linear isomorphisms," Canadian Mathematical Bulletin, vol. 33, no. 2, pp. 139144, 1990.

[10] M. H. Stone, "Applications of the theory of Boolean rings to general topology," Transactions of the American Mathematical Society, vol. 41, no. 3, pp. 375-481, 1937.

[11] F. J. MacWilliams, Combinatorial problems of elementary abelian groups [Ph.D. thesis], Harvard University, 1962.

[12] F. J. MacWilliams, "A theorem on the distribution of weights in a systematic code," The Bell System Technical Journal, vol. 42, pp. 79-94, 1963.
[13] H. Q. Dinh and S. R. López-Permouth, "On the equivalence of codes over rings and modules," Finite Fields and their Applications, vol. 10, no. 4, pp. 615-625, 2004.

[14] H. Gluesing-Luerssen, "On isometries for convolutional codes," Advances in Mathematics of Communications, vol. 3, no. 2, pp. 179-203, 2009.

[15] M. Ferrer, S. Hernandez, and D. Shakhmatov, "Subgroups of direct products closely approximated by direct sums," http://arxiv.org/abs/1306.3954.

[16] G. D. Forney Jr. and M. D. Trott, "The dynamics of group codes: dual abelian group codes and systems," IEEE Transactions on Information Theory, vol. 50, no. 12, pp. 2935-2965, 2004.

[17] J. Rosenthal, J. M. Schumacher, and E. V. York, "On behaviors and convolutional codes," IEEE Transactions on Information Theory, vol. 42, no. 6, pp. 1881-1891, 1996.

[18] J. C. Willems, "From time series to linear systems, parts I-III," Automatica, vol. 22, pp. 561-580, 615-694, 1986.

[19] K. Eda, T. Kiyosawa, and H. Ohta, " $N$-compactness and its applications," in Topics in General Topology, vol. 41, pp. 459-521, North-Holland, Amsterdam, The Amsterdam, 1989.

[20] M. V. Ferrer, S. Hernández, and A. M. Ródenas, "Automatic continuity of biseparating homomorphisms defined between groups of continuous functions," Topology and Its Applications, vol. 157, no. 8, pp. 1395-1403, 2010.

[21] S. Hernández and A. M. Rodenas, "Automatic continuity and representation of group homomorphisms defined between groups of continuous functions," Topology and Its Applications, vol. 154, no. 10, pp. 2089-2098, 2007.

[22] J. Martinez, “C $(X, Z)$ revisited," Advances in Mathematics, vol. 99, no. 2, pp. 152-161, 1993.

[23] H. Ohta, "Chains of strongly non-reflexive dual groups of integer-valued continuous functions," Proceedings of the American Mathematical Society, vol. 124, no. 3, pp. 961-967, 1996.

[24] A. M. Ródenas, Grupos de funciones continuas [Ph.D. thesis], 2006.

[25] J. S. Yang, "Transformation groups of automorphisms of $\mathrm{C}(\mathrm{X}, \mathrm{G})$," Proceedings of the American Mathematical Society, vol. 39, no. 3, pp. 619-624, 1973.

[26] J. S. Yang, "On isomorphic groups and homeomorphic spaces," Proceedings of the American Mathematical Society, vol. 43, pp. 431-438, 1974.

[27] R. Engelking, General Topology, Polish Scientific, Warsaw, Poland, 1977.

[28] A. A. Milutin, "Isomoprhisms of spaces of continuous functions on compacts of power continuum," Teoria Funcionalista, vol. 2, pp. 150-156, 1966 (Russian).

[29] T. Orenshtein and B. Tsaban, "Pointwise convergence of partial functions: the Gerlits-Nagy problem," Advances in Mathematics, vol. 232, pp. 311-326, 2013. 


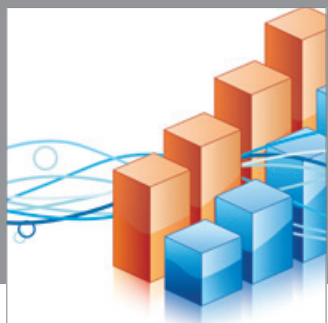

Advances in

Operations Research

mansans

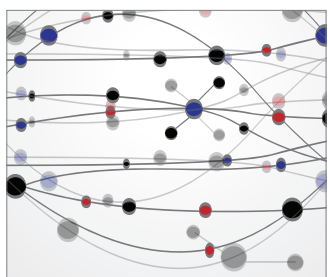

The Scientific World Journal
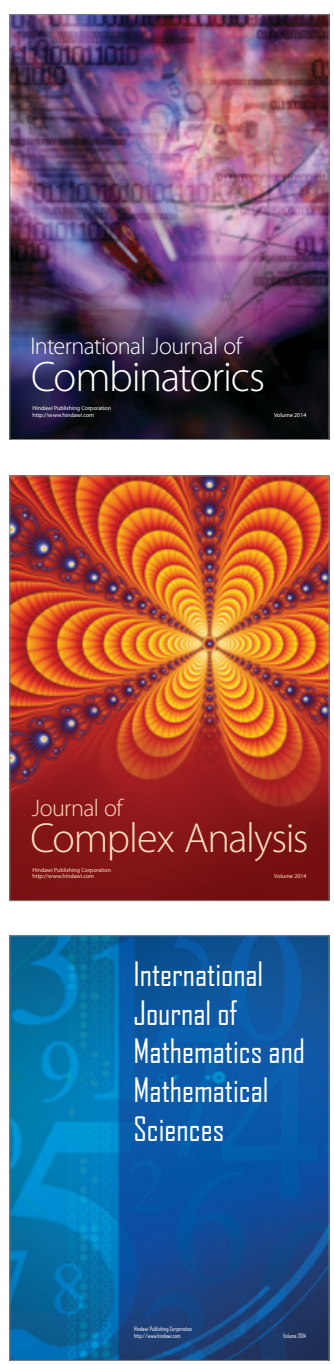
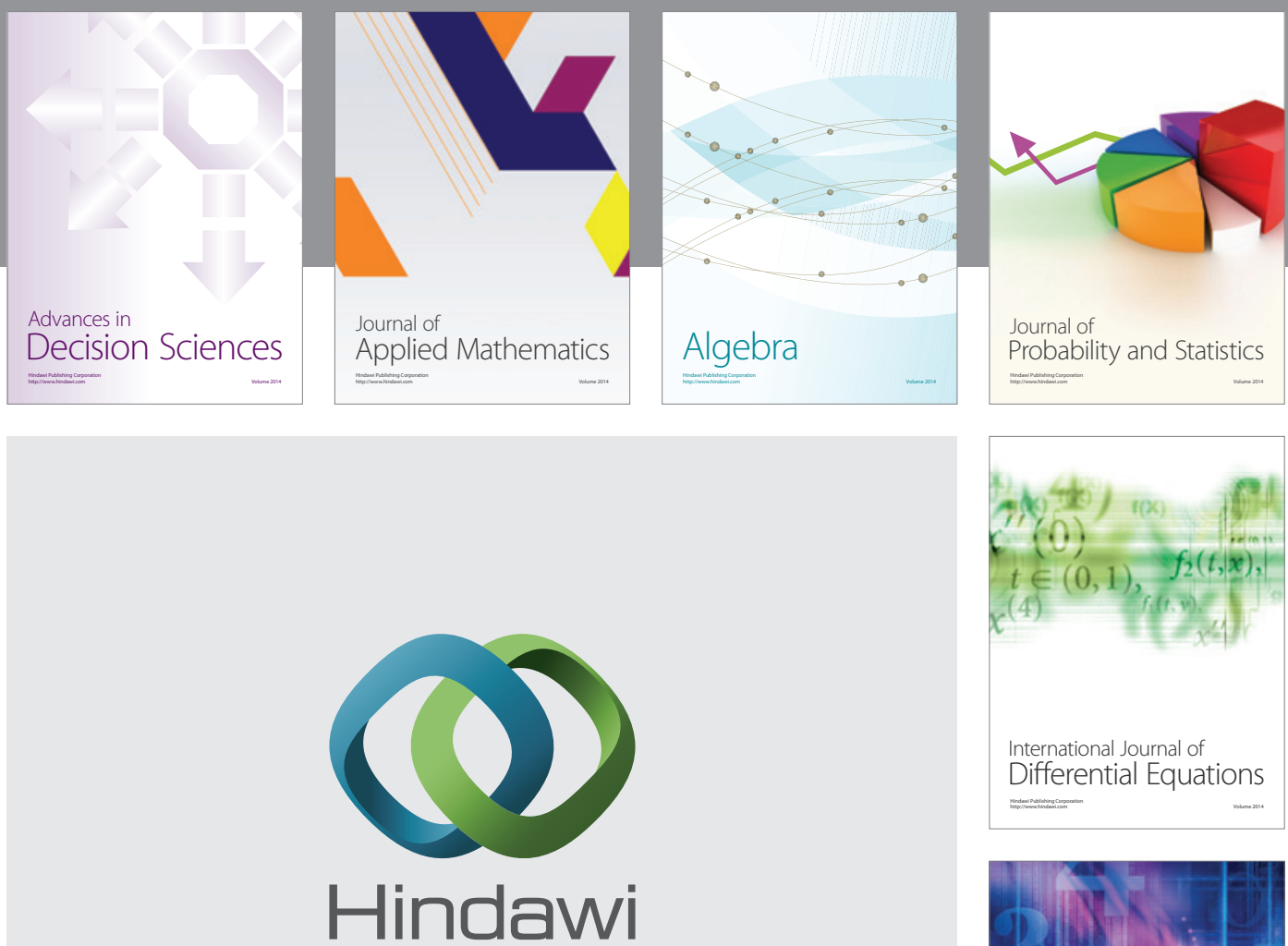

Submit your manuscripts at http://www.hindawi.com
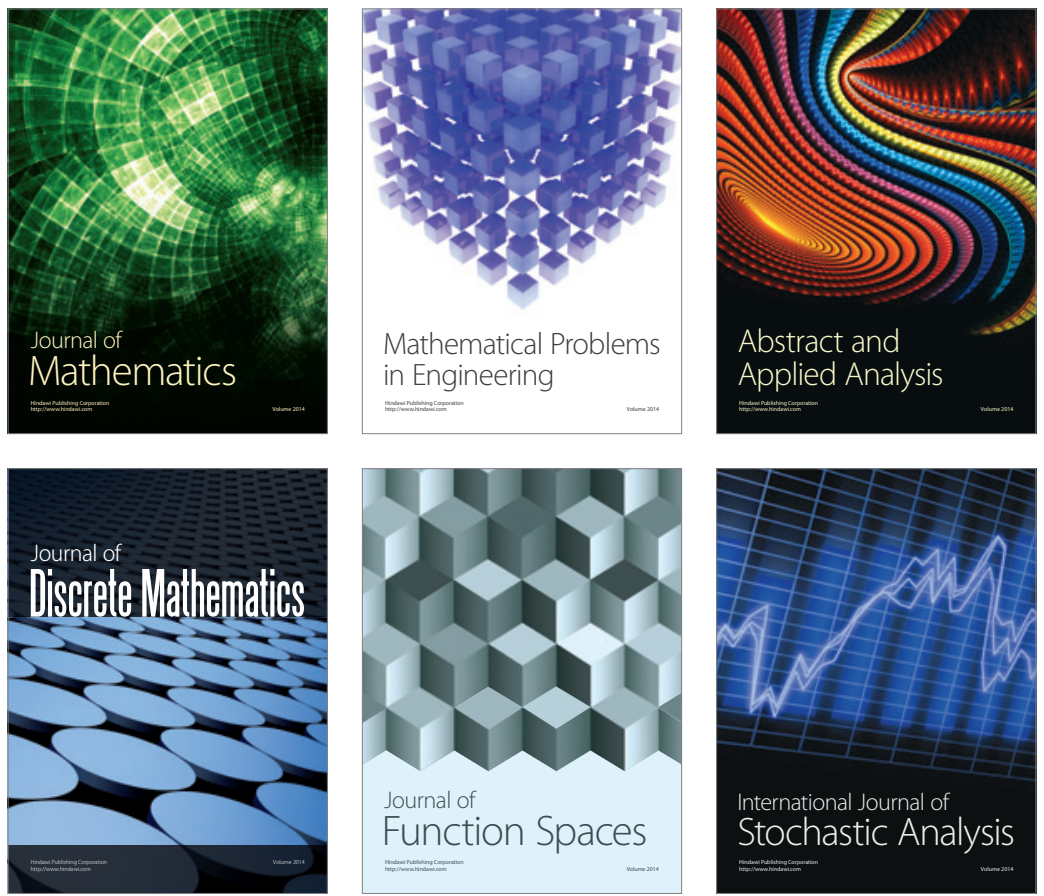

Journal of

Function Spaces

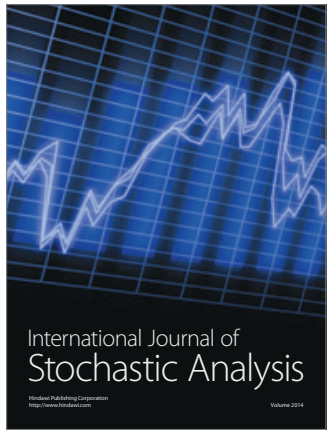

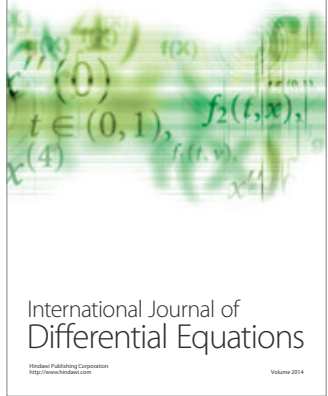
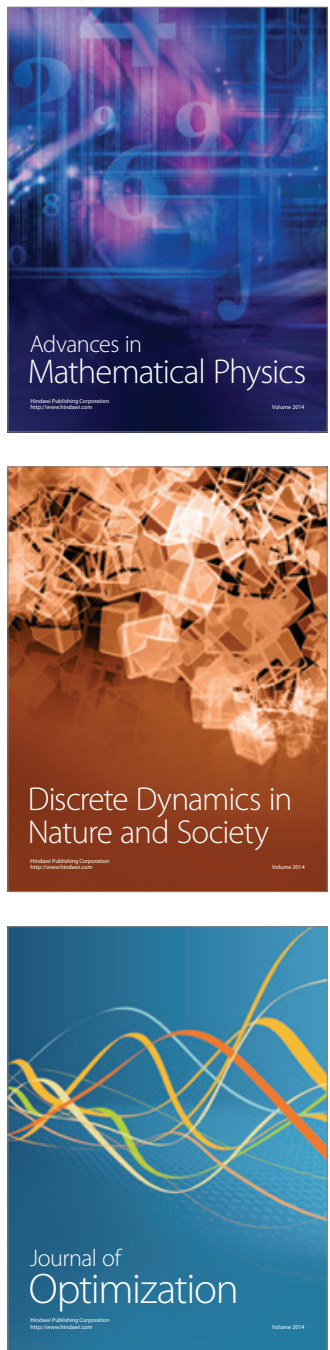